\title{
ON A CONJECTURE OF LIN-NI FOR A SEMILINEAR NEUMANN PROBLEM
}

\author{
ADIMURTHI AND S. L. YADAVA
}

ABstract. Let $\Omega$ be a bounded domain in $\mathbb{R}^{n}(n \geq 3)$ and $\lambda>0$. We consider

$$
\begin{aligned}
-\Delta u+\lambda u & =u^{(n+2) /(n-2)} & & \text { in } \Omega, \\
u & >0 & & \text { in } \Omega, \\
\frac{\partial u}{\partial \nu} & =0 & & \text { on } \partial \Omega,
\end{aligned}
$$

and show that for $\lambda$ sufficiently small, the minimal energy solutions are only constants.

\section{INTRODUCTION}

Let $n \geq 3$ and $\Omega \subset \mathbb{R}^{n}$ be a bounded domain with smooth boundary. For $1<p<\infty$ and $\lambda>0$, we consider the following problem

$$
\begin{cases}-\Delta u+\lambda u=u^{p} & \text { in } \Omega, \\ u>0 & \text { in } \Omega, \\ \frac{\partial u}{\partial \nu}=0 & \text { on } \partial \Omega .\end{cases}
$$

This is the stationary problem for the Keller-Segal [11] system which describe the chemotactic aggregation stage of cellular slim molds (see also Schaaf [16]). Clearly $u=\lambda^{1 /(p-1)}$ is a solution of (1.1). In general the existence of nonconstant solutions depend on $\lambda$ and $p$.

For $p<(n+2) /(n-2)$, problem (1.1) has been discussed by Lin-Ni-Takagi [13]. They showed that there exist positive constants $\lambda_{0}$ and $\lambda_{1}$, with $\lambda_{0} \leq \lambda_{1}$, such that, for $\lambda>\lambda_{1},(1.1)$ admits a nonconstant solution and for $\lambda \leq \lambda_{0},(1.1)$ does not admit any nonconstant solution.

When $\Omega$ is a ball, Ni [14] has shown that for any $p>1$, there exists a $\lambda_{1}>0$, such that, for $\lambda>\lambda_{1},(1.1)$ admits a nonconstant radial solution. In Lin-Ni [12], it has been shown that if $p \neq(n+2) /(n-2)$, there then exists a $\lambda_{0}>0$, such that, for $\lambda<\lambda_{0},(1.1)$ does not admit any radial nonconstant solution. In view of these results, Lin-Ni [12] made the following

Conjecture. For $p>1$, there exist positive constants $\lambda_{0}$ and $\lambda_{1}$, with $\lambda_{0} \leq \lambda_{1}$, such that

Received by the editors December 3, 1990.

1980 Mathematics Subject Classification (1985 Revision). Primary 35J25; Secondary 35P30. 
(a) If $\lambda>\lambda_{1}$, then (1.1) admits a nonconstant solution.

(b) If $\lambda<\lambda_{0}$, then (1.1) does not admit any nonconstant solution.

Now we analyze $(1.1)$ for the critical case $p=(n+2) /(n-2)$. Using the variational techniques, Adimurthi-Mancini [2] (see also X. J. Wang [17]) has shown the existence of a minimal energy solution of (1.1) for every $\lambda>0$. Moreover by comparing the energy of these solutions with that of constant solutions, they obtained a constant $\lambda_{1}>0$, such that, for $\lambda>\lambda_{1}$, the minimal energy solutions are not constant. From this, it follows that part (a) of the conjecture is true in this case. However it is not clear, for $\lambda$ small, that the minimal energy solutions are constants or not.

When $\Omega$ is a ball and $p=(n+2) /(n-2)$, using the shooting argument, it has been shown in Adimurthi-Yadava [3] and Budd-Knaap-Peletier [7] that, if $n \in\{4,5,6\}$, there exists a $\lambda_{0}>0$, such that, for $\lambda<\lambda_{0},(1.1)$ admits nonconstant radial solutions. Note that this gives a counterexample to part (b) of the conjecture.

In view of these results, it is natural to ask that part (b) of the conjecture holds at least for minimal energy solutions when $p=(n+2) /(n-2)$.

In this paper we show that it is indeed true. In order to state our main result, we restate some known results.

Let $b>0$ and define

$$
f(t)= \begin{cases}|t|^{4 /(n-2)} t & \text { if } n \geq 3 \\ h(t) e^{b t^{2}} & \text { if } n=2\end{cases}
$$

where $h(t) e^{b t^{2}}$ is a function of critical growth (see definition (2.1) in [4]). Let $F$ be its primitive given by

$$
F(t)=\int_{0}^{t} f(s) d s .
$$

Let $n \geq 2$ and $\Omega \subset \mathbb{R}^{n}$ be a bounded domain with smooth boundary. For a measurable function $u$ on $\Omega$ and $1 \leq q \leq \infty$, denote

$$
\begin{aligned}
|u|_{q}^{q} & =\int_{\Omega}|u|^{q} d x \quad \text { if } q<\infty, \\
|u|_{\infty} & =\operatorname{ess} \sup \{|u(x)|, x \in \bar{\Omega}\} .
\end{aligned}
$$

For $u \in H^{1}(\Omega)$ and $\lambda>0$, define

$$
\begin{gathered}
\|u\|^{2}=|\nabla u|_{2}^{2}+|u|_{2}^{2}, \\
J_{\lambda}(u)=\frac{1}{2}|\nabla u|_{2}^{2}+\frac{\lambda}{2}|u|_{2}^{2}-\int_{\Omega} F(u) d x \\
\partial B_{\lambda}=\left\{u \in H^{1}(\Omega) \backslash\{0\} ;|\nabla u|_{2}^{2}+\lambda|u|_{2}^{2}=\int_{\Omega} f(u) u d x\right\} \\
\frac{a_{\lambda}^{2}}{2}=\inf \left\{J_{\lambda}(u) ; u \in \partial B_{\lambda}\right\} .
\end{gathered}
$$

For $n \geq 3$, let $S$ denote the best Sobolev constant given by

$$
S=\inf \left\{\int_{\mathbb{R}^{n}}|\nabla u|^{2} d x: \int_{\mathbb{R}^{n}}|u|^{2 n /(n-2)}=1\right\} .
$$


Consider the following problem

$$
\begin{cases}-\Delta u+\lambda u=f(u) & \text { in } \Omega, \\ u>0 & \text { in } \Omega, \\ \frac{\partial u}{\partial \nu}=0 & \text { on } \partial \Omega .\end{cases}
$$

From [2 and 4] we have the following

Theorem 1.1. Let $\lambda>0$ and let $\Omega$ and $a_{\lambda}$ be as above. Then:

(i) If $n \geq 3$, then there exists a solution $u_{\lambda}$ of (1.2) such that

$$
a_{\lambda}^{2} / 2=J_{\lambda}\left(u_{\lambda}\right)<S^{n / 2} / 2 n .
$$

(ii) If $n=2$ and assume further that $f^{\prime}(0)=0$ and

$$
\varlimsup_{t \rightarrow \infty} h(t) t=\infty,
$$

then there exists a solution $u_{\lambda}$ of (1.2) such that

$$
a_{\lambda}^{2} / 2=J_{\lambda}\left(u_{\lambda}\right)<\pi / b .
$$

(iii) Moreover there exists a constant $\lambda_{1}>0$ such that, for $\lambda>\lambda_{1}, u_{\lambda}$ are not constants.

The solutions obtained in the above theorem are called minimal energy solutions. For the proof of this theorem, we refer the following:

(i) and (iii) follows from Theorem 1.2 of Adimurthi-Mancini [2] and (ii) and (iii) follows from Theorem 2.1, Corollary 2.2 and Lemma 3.8 of AdimurthiYadava [4]. Now we state our main result.

Main Theorem. Let $f$ satisfy the hypotheses of Theorem 1.1. Then there exists a positive constant $\lambda_{0}$, such that, for all $\lambda<\lambda_{0}$, the minimal energy solutions of (1.2), given by Theorem 1.1, are constants.

\section{Proof of the Main Theorem}

Let $\varepsilon>0$ and $\mu>0$. Define

$$
\begin{gathered}
A_{\mu}=\{(u, \lambda) ; u \text { satisfies }(1.2) \text { for some } \lambda \leq \mu\}, \\
A_{\mu, \varepsilon}=\left\{(u, \lambda) \in A_{\mu} ; J_{\lambda}(u)<(1-\varepsilon) \pi / b, \text { if } n=2 n\right. \text { and } \\
\left.J_{\lambda}(u)<(\cdot 1-\varepsilon) \frac{S^{n / 2}}{2 n}, \text { if } n \geq 3\right\} .
\end{gathered}
$$

Lemma 2.1. Let $\varepsilon>0, \mu>0$ and $A_{\mu, \varepsilon}$ be as above. Let $\left\{\left(u_{k}, \lambda_{k}\right)\right\} \in A_{\mu, \varepsilon}$ such that $\lambda_{k} \rightarrow 0$ as $k \rightarrow \infty$. Then $\lim _{k \rightarrow \infty}\left|u_{k}\right|_{\infty}=0$.

Proof. Since $\left(u_{k}, \lambda_{k}\right)$ is in $A_{\mu, \varepsilon}$, it follows that $\left\{\left\|u_{k}\right\|\right\}$ is bounded in $H^{1}(\Omega)$ and

$$
\sup _{k} \int_{\Omega} f\left(u_{k}\right) u_{k} d x<\infty
$$

Let $u_{k} \rightarrow u_{0}$ weakly and a.e. in $\Omega$.

Claim $1 . u_{0} \equiv 0$ and $\left\|u_{k}\right\| \rightarrow 0$ as $k \rightarrow \infty$. 
Clearly $u_{0}$ satisfies

$$
\begin{aligned}
-\Delta u_{0} & =f\left(u_{0}\right) & & \text { in } \Omega, \\
u_{0} & \geq 0 & & \text { in } \Omega, \\
\frac{\partial u_{0}}{\partial \nu} & =0 & & \text { on } \partial \Omega
\end{aligned}
$$

and hence $u_{0} \equiv 0$. Therefore by Rellich's lemma $\left|u_{k}\right|_{2} \rightarrow 0$ as $k \rightarrow \infty$. Hence we have

$$
\begin{aligned}
\varlimsup_{k \rightarrow \infty} J_{1}\left(u_{k}\right) & =\varlimsup_{k \rightarrow \infty}\left\{J_{\lambda_{k}}\left(u_{k}\right)+\frac{\left(1-\lambda_{k}\right)}{2}\left|u_{k}\right|_{2}^{2}\right\} \\
& \leq(1-\varepsilon) \begin{cases}\frac{S^{n / 2}}{2 n} & \text { if } n \geq 3, \\
\frac{\pi}{b} & \text { if } n=2 .\end{cases}
\end{aligned}
$$

Let $\omega \in H^{1}(\Omega)$ and if we denote $J_{1}^{\prime}$ is the Fréchet derivative of $J_{1}$, then

$$
\begin{aligned}
\left|\left\langle J_{1}^{\prime}\left(u_{k}\right), \omega\right\rangle\right| & =\left|\left\langle J_{\lambda_{k}}^{\prime}\left(u_{k}\right), \omega\right\rangle+\left(1-\lambda_{k}\right) \int_{\Omega} u_{k} w d x\right| \\
& \leq\left(1-\lambda_{k}\right)\left|u_{k}\right|_{2}\|w\|
\end{aligned}
$$

and hence

$$
\left\|J_{1}^{\prime}\left(u_{k}\right)\right\| \leq\left(1-\lambda_{k}\right)\left|u_{k}\right|_{2} \rightarrow 0
$$

as $k \rightarrow \infty$. Since $J_{1}$ satisfies Palais-Smale condition on $\left(-\infty, S^{n / 2} / 2 n\right)$ if $n \geq 3$ and on $(-\infty, \pi / b)$ if $n=2$ (proof of this follows as in the Dirichlet case. See Brezis-Nirenberg [6], Grossi-Pacella [10] if $n \geq 3$ and Adimurthi [1], Adimurthi-Yadava [5] if $n=2$ ), from (2.4) we can extract a convergent subsequence of $\left\{u_{k}\right\}$. Since $u_{0} \equiv 0$, we obtain $\left\|u_{k}\right\| \rightarrow 0$ as $k \rightarrow \infty$ and this proves the claim.

Claim 2. Let $n \geq 3$, then

$$
\varlimsup_{k \rightarrow \infty}\left|u_{k}\right|_{\infty}<\infty .
$$

Suppose (2.5) is not true. Let $P_{k} \in \bar{\Omega}$ be such that

$$
M_{k}=u_{k}\left(P_{k}\right)=\left|u_{k}\right|_{\infty} \text {. }
$$

Then for a subsequence $M_{k} \rightarrow \infty$ and $P_{k} \rightarrow P_{0}$ as $k \rightarrow \infty$. Let $t_{k}$ be defined by

$$
M_{k} t_{k}^{(n-2) / 2}=1 .
$$

Let $B(z, R)$ denote the open ball of radius $R$ with centre at $z$. For a subsequence, one of the following holds: either

$$
\lim _{k \rightarrow \infty} \frac{d\left(P_{k}, \partial \Omega\right)}{t_{k}}=\infty
$$

or

$$
\lim _{k \rightarrow \infty} \frac{d\left(P_{k}, \partial \Omega\right)}{t_{k}}<\infty .
$$

In case of (2.8), for every $R>0$, we can choose a $k_{0}>0$ such that $B\left(P_{k}, t_{k} R\right) \subset \Omega$ for $k \geq k_{0}$. Let $B_{k}(R)=B_{0}(R)=B(0, R)$. In case of 
(2.9), let $Q_{k} \in \partial \Omega$ such that $d\left(P_{k}, Q_{k}\right)=d\left(P_{k}, \partial \Omega\right)$. Let $\nu_{k}$ be the unit inward normal at $Q_{k}$. Since $\partial \Omega$ is smooth, it satisfies uniformly the inner sphere condition. Therefore, for every $R>0$, we can choose a $k_{0}>0$ such that for $k \geq k_{0}$.

$$
B\left(Z_{k}, t_{k} R\right) \subset \Omega, \quad Z_{k}=P_{k}+R t_{k} \nu_{k} .
$$

Let $B_{k}(R)=B\left(\nu_{k} R, R\right)$ and $B_{0}(R)=B\left(\nu_{0} R, R\right)$ where $\nu_{0}=\lim _{k \rightarrow \infty} \nu_{k}$. For $k>k_{0}$, define $v_{k}$ in $B_{k}(R)$ by

$$
v_{k}(y)=t_{k}^{(n-2) / 2} u_{k}\left(P_{k}+t_{k} y\right) .
$$

Then clearly $v_{k}$ satisfies

$$
\left\{\begin{array}{l}
-\Delta v_{k}=v_{k}^{(n+2) /(n-2)}-\lambda_{k} t_{k}^{2} v_{k} \quad \text { in } B_{k}(R), \\
v_{k}(0)=1, \quad 0<v_{k} \leq 1 .
\end{array}\right.
$$

Therefore by elliptic regularity (see [9]) we have, for every $0<\alpha<1$,

$$
\varlimsup_{k \rightarrow \infty}\left|v_{k}\right|_{C^{1, \alpha}\left(\overline{\left.B_{k}(R)\right)}\right.}<\infty .
$$

Let $v_{k} \rightarrow v_{0}$ in $C^{1}\left(\overline{B_{0}(R)}\right)$. Then from (2.12) and (2.13), $v_{0}$ satisfies

$$
\left\{\begin{array}{l}
-\Delta v_{0}=v_{0}^{(n+2) /(n-2)} \text { in } B_{0}(R), \\
v_{0} \geq 0, \quad v_{0}(0)=1 .
\end{array}\right.
$$

On the other hand, from Claim 1, we have

$$
\int_{B_{k}(R)}\left|\nabla v_{k}\right|^{2} d y \leq \int_{\Omega}\left|\nabla u_{k}\right|^{2} d x \leq\left\|u_{k}\right\|^{2} \rightarrow 0
$$

as $k \rightarrow \infty$. Hence $\nabla v_{0} \equiv 0$. Since $v_{0}(0)=1$ implies that $v_{0} \equiv 1$, and this contradicts (2.14). This proves the Claim 2.

Let $n=2$. From Claim 1, $\left\|u_{k}\right\| \rightarrow 0$. Therefore from Cherrier [8] we obtain that, for any $p>1,\left\{\left|f\left(u_{k}\right)\right|_{p}\right\}$ is uniformly bounded. Let $n \geq 3$, then from Claim 2, it follows again that for any $p>1,\left\{\left|f\left(u_{k}\right)\right|_{p}\right\}$ is uniformly bounded. Hence from the regularity of elliptic equations $\left\{\left\|u_{k}\right\|_{w^{2, p}(\Omega)}\right\}$ is bounded and therefore by Sobolev imbedding we have for any $0<\alpha<1$,

$$
\varlimsup_{k \rightarrow \infty}\left|u_{k}\right|_{C^{1, \alpha}(\bar{\Omega})}<\infty .
$$

Since $\left\|u_{k}\right\| \rightarrow 0$, from Arzela-Ascoli's theorem $\lim _{k \rightarrow \infty}\left|u_{k}\right|_{\infty}=0$. This proves the lemma.

Lemma 2.2. Let $\varepsilon>0$ and $\mu>0$. Define

$$
M_{\mu}=\operatorname{Sup}\left\{|u|_{\infty} ; \text { for some } \lambda,(u, \lambda) \in A_{\mu, \varepsilon}\right\}
$$

then

$$
\lim _{\mu \rightarrow 0} M_{\mu}=0 .
$$

Proof. Suppose (2.16) does not hold. Then there exists a sequence $\left(u_{k}, \lambda_{k}\right) \in$ $A_{\lambda_{k}, \varepsilon}$ with $\lambda_{k} \rightarrow 0$ as $k \rightarrow \infty$ and $\lim _{k \rightarrow \infty}\left|u_{k}\right|_{\infty}>0$. This is a contradiction to Lemma 2.1. This proves (2.16). 
Lemma 2.3. Let $\varepsilon>0$. Then there exists $a \mu_{0}>0$ such that $A_{\mu_{0}, \varepsilon}$ consists of constants only.

Proof. Proof of this lemma follows exactly as in Ni-Takagi [15, Theorem 3]. For the sake of completeness we will reproduce their proof. From Poincarés inequality, there exists a $\nu>0$ such that

$$
\nu \int_{\Omega} \varphi^{2} d x \leq \int_{\Omega}|\nabla \varphi|^{2} d x
$$

for all $\varphi \in H^{1}(\Omega)$ with $\int_{\Omega} \varphi d x=0$.

From Lemma 2.2 and using $f^{\prime}(0)=0$, we can choose a $\mu_{0}>0$ such that

$$
f^{\prime}\left(M_{\mu_{0}}\right) \leq \nu / 2 \text {. }
$$

Let $(u, \lambda) \in A_{\mu_{0}, \varepsilon}$, and decompose, $u=u_{0}+\varphi$ where

$$
u_{0}=\frac{1}{|\Omega|} \int_{\Omega} u d x, \quad \int_{\Omega} \varphi d x=0 .
$$

Then $\varphi$ satisfies

$$
\begin{aligned}
-\Delta \varphi+\lambda \varphi & =\rho \varphi+f\left(u_{0}\right)-\lambda u_{0} & & \text { in } \Omega, \\
\frac{\partial \varphi}{\partial \nu} & =0 & & \text { on } \partial \Omega,
\end{aligned}
$$

where $\rho=\int_{0}^{1} f^{\prime}\left(u_{0}+t \varphi\right) d t$.

Since $0 \leq u_{0}+t \varphi \leq u_{0}+\varphi=u \leq M_{\mu_{0}}$, we obtain

$$
|\rho| \leq f^{\prime}\left(M_{\mu_{0}}\right) \leq \nu / 2 \text {. }
$$

From (2.19), (2.20) and (2.17) we have

$$
\begin{aligned}
(\nu+\lambda) \int_{\Omega} \varphi^{2} d x & \leq \int_{\Omega}|\nabla \varphi|^{2} d x+\lambda \int_{\Omega} \varphi^{2} d x \\
& =\int_{\Omega} \rho \varphi^{2} d x \leq \frac{\nu}{2} \int_{\Omega} \varphi^{2} d x .
\end{aligned}
$$

This implies that $\varphi \equiv 0$ and hence $u$ is a constant. This proves the lemma.

Proof of the Main Theorem. For $\lambda>0$, the constant solution $v_{\lambda}$ of (1.2) is given by

$$
f\left(v_{\lambda}\right) / v_{\lambda}=\lambda .
$$

Since $f^{\prime}(0)=0, v_{\lambda}$ exists and tends to zero as $\lambda \rightarrow 0$. Therefore we can choose $\mu_{1}>0$, such that, for all $\lambda \leq \mu_{1}$,

$$
J_{\lambda}\left(v_{\lambda}\right) \leq \begin{cases}S^{n / 2} / 4 n & \text { if } n \geq 3, \\ \pi / 2 b & \text { if } n=2 .\end{cases}
$$

Let $\varepsilon=\frac{1}{2}, \mu_{0}$ is determined as in Lemma 2.3, and $\lambda_{0}=\min \left(\mu_{0}, \mu_{1}\right)$. Let $\lambda<\lambda_{0}$ and $u_{\lambda}$ be a minimal energy solution. Since $J_{\lambda}\left(u_{\lambda}\right) \leq J_{\lambda}\left(v_{\lambda}\right)$, from (2.22), $\left(u_{\lambda}, \lambda\right) \in A_{\lambda_{0}, \varepsilon}$ and hence from Lemma 2.3, $u_{\lambda}$ is constant. This proves the theorem.

\section{ACKNOWLEDGMENT}

The authors thank the referee for pointing out an error in the earlier version of this paper. 


\section{REFERENCES}

1. Adimurthi, Existence of a positive solution of the semilinear Dirichlet problem with critical growth for the n-Laplacian, Ann. Scuola Norm. Sup. Pisa Cl. Sci. 17 (1990), 393-413.

2. Adimurthi and G. Mancini, The Neumann problem for elliptic equations with critical nonlinearity. A tribute in honour of G. Prodi, edited by A. Ambrosetti et al., Scuola Norm. Sup., Pisa, 1991, pp. 9-25.

3. Adimurthi and S. L. Yadava, Existence and non existence of positive radial solutions of Neumann problem with critical Sobolev exponents, Arch. Rational Mech. Anal. 115 (1991), 275-296.

4. _ Critical exponent problem in $\mathbb{R}^{2}$ with Neumann boundary condition, Comm. Partial Differential Equations 15 (1990), 461-501.

5. __ Semilinear elliptic mixed boundary value problem with critical exponent in $\mathbb{R}^{2}$, preprint, 1989.

6. H. Brezis and L. Nirenberg, Positive solutions for a nonlinear elliptic equation involving critical Sobolev exponents, Comm. Pure Appl. Math. 36 (1983), 437-477.

7. C. Budd, M. C. Knaap and L. A. Peletier, Asymptotic behaviour of solutions of elliptic equations with critical exponents and Neumann boundary conditions, Proc. Roy. Soc. Edinburgh 117 (1991), 225-250.

8. P. Cherrier, Meilleures constantes dans des inéqalités relatives aux espaces de Sobolev, Bull. Sci. Math. (2) 108 (1984), 225-262.

9. D. Gilbarg and N. S. Trudinger, Elliptic partial differential equations of second order, Springer-Verlag, New York and Berlin, 1977.

10. M. Grossi and F. Pacella, Positive solutions of nonlinear elliptic equations with critical Sobolev exponent and mixed boundary conditions, Proc. Roy. Soc. Edinburgh 116 (1990), 23-43.

11. E. F. Keller and L. A. Segal, Initiation of slime mold aggregation viewed as an instability, J. Theoret. Biol. 26 (1970), 399-415.

12. C. S. Lin and W. M. Ni, On the diffusion coefficient of a semilinear Neumann problem, Lecture Notes in Math., vol. 1340, Springer, 1986, pp. 160-174.

13. C. S. Lin, W. M. Ni and I. Takagi, Large amplitude stationary solutions to a chemotaxis system, J. Differential Equations 72 (1988), 1-27.

14. W. M. Ni, On the positive radial solutions of some semi-linear elliptic equations on $\mathbb{R}^{n}$, Appl. Math. Optim. 9 (1983), 373-380.

15. W. M. Ni and I. Takagi, On the Neumann problem for some semilinear elliptic equations and systems of activator-inhibitor type, Trans. Amer. Math. Soc. 297 (1986), 351-368.

16. R. Schaaf, Stationary solutions of chemotaxis systems, Trans. Amer. Math. Soc. 292 (1985), 531-556.

17. X. J. Wang, Neumann problem of semilinear elliptic equations involving critical Sobolev exponents, J. Differential Equations 93 (1991), 283-310.

T. I. F. R. Centre, P.B. No. 1234, Bangalore 560 012, India

E-mail address: aditi@tifrbng.ernet.in

E-mail address: yadava@tifrbng.ernet.in 